\title{
CREGG \\ Commission exploration fonctionnelle
}

\author{
M. GREFF \\ Institut Arnault Tzanck (Saint-Laurent-du-Var)
}

La Commission Exploration Fonctionnelle du CREGG a atteint les objectifs fixés pour l'année 1991.

En premier lieu nous avons organisé une journée sur la pH-métrie le 6 avril dernier à Paris avec l'aide des Laboratoires Janssen. Cette journée réservée à 20 membres du CREGG a permis un échange fructueux et convivial entre les participants et les orateurs du CREGG connus au niveau international.

Les lettres d'encouragements qui nous sont parvenues nous incitent à proposer pour 1992 une journée sur la manométrie anorectale, thème sensible sur des aspects très divers à savoir les indications vues par le gastroentérologue et le chirurgien, la technique, le matériel, la thérapeutique médicale, chirurgicale ou de rééducation.

En second lieu, nous avons distribué aux membres du CREGG les fiches techniques concernant le matériel disponible à l'heure actuelle en France et les fịches concernant les principaux examens réalisés en exploration fonctionnelle digestive.

L'exploration fonctionnelle est ouverte à tout membre ayant des projets de travail avec des propositions concrètes.

Je tiens à remercier les Docteurs Cargill et Guerder pour leur travail dans la rédaction des fiches, les Docteurs Dupin, Lunaud, Fabre, Congard, et de nouveau les Docteurs Cargill et Guerder pour leur travail lors de la journée sur la $\mathrm{pH}$-métrie.

\section{MEMBRES DE LA COMMISSION EXPLORATION FONCTIONNELLE 1991}

Docteur GREFF, Saint-Laurent-du-Var.

Docteur CARGILL, Paris.

Docteur GUERDER, Toulon.

Docteur DUPIN-NIZARD, Marseille.

Docteur LUNAUD, Clermont-Ferrand.

Docteur PFEIFFER, Colmar.
Docteur SIMON, Nantes.

Docteur POMMELET, Lille.

Docteur TOURNUT, Marseille.

Docteur FABRE, Toulouse.

Docteur CONGARD, Caen.

Docteur YOUSFI, Montpellier. 
Sonde à microcapteurs électroniques de type Kœnigsberg

SOLAL 2 rue du Travail 67000 STRASBOURG Tel 88328985 Fax 88751713

Descriptlf :

Sonde à 3 capteurs électroniques avec capteur à l'antimoine distal sur connecteur ITT CANNON et lumière pour Bernstein. Version ESP à 4 capteurs (l'un à l'extrémité). Disposition des capteurs pouvant être adaptée aux besoins

Date d'apparition sur le marché : 1980

Prix : 34000 F HT (mai 90) pour le modèle 3 capteurs et 37000 F HT pour 4 capteurs

Délal de fourniture : 8 semaines

SAV et garantie : 1 an SAV: prêt d'une sonde durant la période de garantie, au delà réparation sur devis

Nature : Capteur à membrane

Utillsation prévue : Manométrie oesophagienne

Type de signal analysé : pression (analogique)

Caractéristiques physiques : Taille $3,18 \mathrm{~mm}$ (extrémité) et 4,65 (corps) Longueur : $125 \mathrm{~cm}$

Durée de vle : Plusieurs années

Gamme de mesure : $0-300 \mathrm{mmHg}$ capacité de surcharge jusqu'à $1000 \mathrm{mmHg}$

Entretlen conselllé : Simple nettoyage à l'eau ou à l'alcool. Stérilisation au glutaraldéhyde ou oxyde d'éthyle. Eviter le chauffage (destruction de la sonde). Fiche d'entretien fournie avec le cathéter.

\section{Commentalre technique :}

Capteur de pression interessant par sa vitesse de réponse, par sa souplesse, qui semble être d'une solidité acceptable. Il permet de s'affranchir de la perfusion, peut être employé chez l'enfant. La résolution est bonne. La capacité de surcharge le met pratiquement à l'abri de tout accident. Bonne linéarité (士 $1 \%)$. Peut être rendu compatible avec de nombreux matériels. Bonne isolation des circuits. Procédure d'étalonnage (sur appareil Sandhill TDS) agéable et séduisante

\section{Commentaire de I'Expert CREGG}

Capteur utilisant une technologie interessante restant de prix élevé, à intégrer dans une chaîne performante

afin d'en uiliser toutes les possibilités. Sa vitesse de réponse premet des études sphinctériennes supérieures plus précises qu'avec les catheters perfusés. Utilisé chez l'adulte comme chez l'enfant sans problème particulier. Le capteur à l'antimoine distal ne nous est pas apparu comme indispensable.

Commentalre général de la Commission :

Périphérique de bonne qualité aux possibilités interessantes dont la vitesse de réponse permet de bonnes études pharyngo-sphinctériennes. Les seuls reproches restent le prix élevé qui fait réfléchir à l'acquisition de ce type de sonde et la non adaptation à l'ensemble des matériels. Outre l'utilisation sur matériel Sandhill (évidente) il est possible de l'utiliser sur Beckman Sensormedics et sur Honeywell. Pour les autres marques des adaptations plus ou moins complexes sont parfois réalisables 


\section{PERSONAL PERINEOMETER}

SOLAL 2 rue du Travail 67000 STRASBOURG

Tel $88 \quad 328985 \quad$ Fax $88 \quad 75 \quad 17 \quad 13$

Descriptif :

Périnéomètre pour biofeedback à domicile des muscles releveurs utilisant le signal éléctromyographique périnéal et le traduisant sous une forme lumineuse et sonore

Date d'aparition sur le marché : 1990

\section{Prix : NON FOURNI}

Capteur conselllé : Vaginaux et rectaux, aldultes et pédiatriques en acrylique ou en ébonite avec électrodes en argent

Enregistreur Type convertisseur analogique-numérique transportable 1 voie, 5 marqueurs d'événement, logiciel et imprimante intégrés sous forme d'une platine séparée (mais sortie PC compatible prévue), une acquisition toutes les 2 secondes

Durée de vle : plusieurs années

Configuration : fixe

Signal digitallsé : nombre de Bits non précisé à $0,5 \mathrm{~Hz}$

Mobllité - Tallle - Polds : $90^{\star} 80^{\star} 200 \mathrm{~mm}$ - $450 \mathrm{~g}$

Coût de fontionnement: Accus rechargeables - Capteurs

Système d'analyse :

Le signal EMG global du périnée est amplifié ( 3 échalles $0-5$ microvolts, $0-10$ et $0-20$ ) et traduit sous une forme lumineuse (ou lumineuse et sonore) pour adaptation du travail par le patient. La fréquence des périodes de travail est adaptable.

Entretlen conselllé:

Désinfection au glutaraldẹhyde ou équivalent des capteurs. Recharge fréquente des accus

Commentalre technlque:

Appareil récent pouvant être interessant pour la prise en charge de certains périnées descendants.

\section{Commentalre de l'Expert CREGG}

Appareil adopté immédiatement par la plupart des maldes. Mais son prix élevé (non communiqué dans la fiche technique) le rend inaccessible à la plupart des patient et ce d'autant qu'il n'existe pas de prise en charge. Les jeux de sondes adaptés a l'âge, permettant pour certaines le passage de cathéters, sont simple à utiliser. Un piège cependant: la contraction abdominale est percue comme un signal positif d'ou un effet parfois pervers de l'utilisation. A notre sens à plus sa place dans certains cabinets spécialisés que chez le malade.

Commentalre général de la Commission :

Appareil interessant qui pourrait être amélioré par l'usage de capteurs abdominaux associés et dont le prix reste trop élevé pour un véritable usage simple à domicile. 
Infuseur pneumo-hydraulique

SOLAL 2 rue du Travail 67000 STRASBOURG

Tel. 88328985

Fax: $887517 \quad 13$

Descrlptlf :

Infuseur pneumohydraulique 4 à 8 canaux à catheters perfusés existant en deux versions: l'une avec une bouteille d'azote, l'autre avec une pompe électrique

Date d'apparltion sur le marché : >20ans

Prix : pour 4 voies HT 30 000F à compresseur électrique, $34000 \mathrm{~F}$ à pression d'azote

Délal de fourniture : 4 à 6 semaines

SAV et garantle : 1 an, SAV par échange standard des pièces défectueuses

Entretlen : Aucun entretien particulier dans les conditions habituelles d'utilisation

Coût d'utllisation : Fonction de l'importance de l'utilisation. Eau distillée, bouteille d'Azote ou courant électrique

Type :

Infuseur pneumo-hydraulique basse compliance pour perfusion de cathéters de manométrie

Commentaire technique:

Matériel intéressant par sa réserve d'eau relativement importante (1,5litres ) avec niveau d'eau visible, permettant soit des examens longs (manométrie du grêle), soit une succession d'examens brefs sans aucune manipulation. Les pieds sont de niveau règlable. Les cathéters en téflons sont agréables, fiables. L'utilisation du compresseur s'est avérée aussi fiable que celle de l'azote. Dans cette configuration la pression dans le réservoir est adaptée par le compresseur dès qu'elle chute un peu. Malgré l'utilisation d'air peu de bulles sont observées dans le circuit totalement transparent facile à surveiller.

\section{Commentalre de l'Expert CREGG}

Aisément utilisable pour toutes sortes d'examens en raison de son pré-équipement en 10 voies. Cette pompe mérite mérite une attention toute particulière par sa souplesse d'emploi, en raison d'un circuit de distribution transparent (mais peut-être pour cette raison un peu fragile) et en raison de cathéters téflon solides, faciles à entretenir. Utilisables avec tous types de capteurs de pression.

Commentaire général de la Commlssion :

Pompe de bonne facture, un peu plus encombrante que certaines, de prix attractif, pratique et simple à utiliser peut être un peu moins connue que l'infuseur Arndorfer mais soutenant largement la comparaison. 
M 60

LTI Groupe GIMED 31 rue Raymond Aron 76130 MONT SAINT AIGNAN

Tel (1) 48780138

Descriptlf :

Appareil d'acquisition et de traitement du signal dans l'exploration foctionnelle manométrique (œsophage - rectum - possibilité d'Urodynamique)

Date d'aparitlon sur le marché : 1989

Prix : 3 voies 100 000F TTC 4 voies 110000 TTC prix début 1990

Délal de fourniture : 5 semaines

SAV et garantle : é change standard des modules sous 48 heures

Garantle : Garantie 2 ans Pièces \& MO (informatique 1 an)

Contrat évolutif : oui gratuit pour la partie logiciel

Capteur conselllé : pont de jauge (capteur de pression) $0+5 \mathrm{~V}$ intégrés et isolés

Enreglstreur Type thermique optionnel 4 à 8 voies

Durée de vle : plusieurs années

Pérlphériques: Capteurs de pression (inclus), Télécommande (micro-soft), Enregistreur, sondes de manométrie et capteurs de déglutition (EMG)

Configuration : modulable par informatique interne

Signal digitalisé : nombre de Bits 12 acquis à $40 \mathrm{~Hz}$ voie par voie \& $200 \mathrm{~Hz}$ à plein usage par microproceseur 80286 type IBM PC $12 \mathrm{MHz}$, multiplexage

Mobllité - Tallle - Poids : $1000 \mathrm{~mm}^{*} 1000 \mathrm{~mm} * 600 \mathrm{~mm} 15 \mathrm{~kg}$ sur roulettes

UtIllsation autres que Gastro-entérologle: UROLOGIE

Type de signaux analysés:

Computer nécessaire: PC compatible

Typres de signaux analysés: Pressions, déglutition, EMG

Analyse point par point à vitesse maximum (partie traitement \& acquisition communes)

Coût de fontlonnement: Négligeable (papier standard A4, encre) plus élevé si utilisation d'une imprimante thermique

Type de sauvegarde des résultats :

Disquette ( 1 à 5 examen) ou disque dur ( 60 à 200 examens) trace papier (si enregistreur)

Tallle loglciel: $3 * 200 \mathrm{KO}$

Langage utillsé : TURBO C

Imprimante conselllée : tous type imprimante compatible PC (laser, jet d'encre, 24 aiguilles au moins) 


\section{Commentalre technlque :}

Appareil français récent facile à manipuler incluant d'emblée un compuler ce qui rend ce matériel particulièrement cohérent, d'une mise en ceuvre rapide. L'utilisation d'une télécommande permet de manipuler l'ordinateur tout en restant près du patient. L'ensemble est livré avec une table roulante sur laquelle est installée le computer et ses périphériques (ainsi qu'avec une chaise!). Le programme est très convivial et même un néophyte en informatique y retrouve vite son chemin. La vitesse de saisie est excellente et les courbes sont identiques à celles obtenue sur un enregistreur de type Beckman. A noter l'adjonction possible d'une imprimante thermique capable de restituer un tracé de très bonne qualité. L'informatique fournie semble de bonne tenue et chauffe moins que certains matériels courament utilisés. Le signal est heureusement digitalisé sur 12 Bits ce qui garanti le bon respect des valeurs mesurée. Le type de langage utilisé permet une analyse rapide que le logiciel rend souple et aisée. Notons enfin que les têtes de pression ont une bonne linéarité, quelles admettent des capacités de surcharges considérables ce qui leur garanti une longue durée de vie.

\section{Commentaire de l'Expert CREGG}

Appareil récent cohérent interessant tant sur les capacités de son informatique que sur celles de la mesure du signal où par son prix attractif. II est fourni comme une véritable station de travail autonome et prête à l'emploi. Le logiciel et les techniques employés sont sans défaut majeur sans doute un des læder du moment.

Commentaire général de la Commission :

Appareil de conception moderne et séduisante utilisant une informatique performante et souple. Ce matériel concu et construit en france (y compris pour la partie informatique) supporte la comparaison avec les leaders du marché 
Sondes manométriques

SOLAL 2 rue du Travail 67000 STRASBOURG

Tól : 88328985

Fax : 88751713

Descriptlf :

Sondes manométriques en polyvinyle transparent extrudé avec repères radio-opaques.

- Manométrie oesophagienne avec et sans ballonnet

- Manométrie du grêle el du colon

- Manométrie ano-rectale

Caractéristlques :

Différentes selon les sondes (cf catalogue sur demande), non fournies par le constructeur. Prix variant de 900 TTC (environ pour un cathéter pédiatrique) à $3000 \mathrm{~F}$ (environ pour une sonde de profilométrie rectale ou pour étude de lintestin grêle). Fabrication de sondes sur mesure possible.

Commentalre technique :

Se rapporter au catalogue selon le type d'analyse désirée

Commentalre de l'Expert CREGG

Un des plus anciens concepteurs de sondes pour la manométrie (depuis plus de 20 ans!) qu'elle soit oesophagienne, du grêle ou du rectum. Réalise des sondes de bonne facture. Un classsique du genre

Commentalre général de la Commission :

Sondes de bonne facture en ce qui concerne le cathéter perfusé. Nombreux modèles au catalogue. Fabricant suivant de près l'actualité de la motricité digestive et adaptant son catalogue aux tendances modernes 


\section{LACTOSCREEN H2 BREATH TESTER}

SOLAL 2 rue du Travail 67000 STRASBOURG

Tel $88 \begin{array}{llllllll}32 & 89 & 85 & \text { Fax } 88 & 75 & 17 & 13\end{array}$

Descriptif :

Console de mesure de l'hydrogène expiré pour réalisation de breath test

Date d'aparition sur le marché : 1990

Prix : NON FOURNI

Capteur conselllé : inclus dans le système

Enreglstreur pas d'enregistrement des résultats, mesures affichées

Durée de vle : plusieurs années

Configuration : fixe

Mobillté - Tallle - Polds : $370^{*} 180^{*} 250 \mathrm{~mm} \quad 7,5 \mathrm{Kg}$

Coût de fontionnement: Embouts usage unique, Gel silica, matériel pour échantillonnage

Système d'analyse :

Analyse de l'hydrogène expiré pour mesure du temps de transit oro-cæacal et pour détection des pullullation microbiennes intestinales

Entretlen conselllé:

Etalonnage hebdomadaire et nettoyage-stérilisation au glutaraldéhyde des accessoires réutilisables (masques ou embouts).

Commentalre technique :

Appareil d'utilsation simple, adapté à tous les âges. N'exige qu'un minimum de manipulations. Un enregistreur papier et/ou une connexion vers une informatique auraient pu être interessants.

Commentaire de l'Expert CREGG

Appareil non essayé par la commission et semblant être relativement plus simple à utiliser que d'autres. Il reste d'utilisation beaucoup plus rare que d'autres appareils d'explorations et son emploi est limité à quelques centres très spécialisés.

Commentalre général de la Commlssion :

Appareil interessant non essayé par la commission, semblant d'emploi simple. Pas de précision concernant le prix, la garantie, le SAV. 


\section{RMS-P SANDHILL}

SOLAL 2 rue du Travail 67000 STRASBOURG

Tel $88328985 \quad$ Fax $88 \quad 7517 \quad 13$

Descrlptif :

$\mathrm{pH}$-métre portable autonome, 1 à 3 voies, exploitation directe sur imprimante et/ou PC compatible

Date d'apparition sur le marché : 1989

Prix : 26300 F HT (1 voie) prix Avril 1991

Délal de fournlture : 4 semaines

SAV et garantle: Assistance téléphonique, réparation sous 4 jours ou prêt d'appareil

Garantle : Un an pièces et main d'oeuvre

Contrat évolutif : oui

Capteur consellié : Electrode de verre type INGOLD 440 (voire fiche spécifique) durée de vie >> $1000 \mathrm{H}$ ou antimoine de Koenigsberg ou ZYNETICS

Enregistreur Type convertisseur analogique-numérique transportable 1 à 3 voies

Durée de vie : plusieurs années

Conflguration : modulable par informatique interne

Signal digitalisé : nombre de Bits non précisé à 0,5 à $0,1 \mathrm{~Hz}$ programmable

Mobllité - Tallle - Polds : 450 Grammes, $85^{*} 180^{*} 25 \mathrm{~mm}$, ambulatoire

Coût de fontlonnement: 1 pile $9 \mathrm{~V}$ alcaline par examen

Système d'analyse :

Computer nécessaire : informatique embarquée dans le pH-mètre ou PC compatible

Type de signaux analysés $\mathrm{pH}$

Configuration modulable

Type de sauvegarde des résultats :

Trace papier, ou support magnétique informatique

Tallle loglciel: 3 Mégabytes

Langage utillsé : "quick basic" (Microsoft compiled basic)

Imprimante conselllée : EPSON LX 800 (liaison par connecteur optique sur sortie RS232) 


\section{Commentalre technique :}

Appareil très récent facile à programmer avec un logiciel embarqué bilingue français anglais. Permet la pH-métrie à plusieurs niveaux. La reproduction directe sur papier est de bonne qualité. La présence d'un boitier métallique met l'appareil à l'abri de certaines perturbations. La version avec analyse sur micro-ordinateur permet une analyse très détaillée. La fréquence théorique d'interrogation de la courbe peut étre de 1 point toutes les 2 secondes (sur une seule voie) ce qui est interessant. La taille mémoire reste un peu limitée pour les examens en multivoies. Poids un peu limite pour l'utilisation pédiatrique. Une innovation proposée mais non essayée par la commission : une sonde à l'antimoine avec référence incorporée. Le choix d'une sonde à l'antimoine ou d'une de verre est à déterminer avant l'achat (préamplificateur différent). De plus on peut utiliser au choix un ou plusieurs marqueurs, la position debout ou couchée est au choix automatique ou manuelle. A noter l'intéressante possibilité de changer les accus sans éffacer la mémoire.

\section{Commentalre de l'Expert CREGG}

Appareil récent à suivre. Des capacités qui ne semblent pas inintéressantes.

Commentalre général de la Commlssion :

Appareil à priori intéressant mais manque de recul pour tirer des conclusions définitives tant sur le plan technologique que commercial. 
PH 60

LTI Groupe Gimed 9 rue Buffault 75009 PARIS

Tel: 48780138

Descriptif :

pH-mètre portable autonome,2 voies, exploitation directe sur imprimante soit sur micro-ordinateur PC compatible ou Macintosh

Date d'apparition sur le marché : 1988

Prlx : 36000 F TTC prix Mai 90

Délal de fournlture : 3 semaines

SAV et garantle : Garantie 2 ans pièces et main d'œuvre hors informatique, échange standard

Contrat évolutlf : gratuit pour la partie logicielle

Capteur conselllé : Electrode de verre type INGOLD 440 (voire fiche spécifique) durée de vie >> $1000 \mathrm{H}$

Enreglstreur Type convertisseur analogique-numérique transportable , mémoire vive , 2 voies

Durée de vle : plusieurs années

Conflguration : modulable par informatique interne

Signal digltallsé : sur 8 Bits 0,25 à $1 \mathrm{~Hz}$

Mobllité - Tallle - Polds : 500 Grammes, $90^{*} 155^{\star} 42 \mathrm{~mm}$, ambulatoire

Coût de fonctionnement: négligeable : fonctionne sur accus rechargeables

Système d'analyse :

Computer nécessaire : informatique embarquée dans le pH-mètre (si restitution sur imprimante).

Micro ordinateur PC compatible ou Macintosh

Type de signaux analysés pH ou ddp

Configuration modúlable

Type de sauvegarde des résultats :

Trace papier(imprimante) Disquette (de 16 à 63 examen suivant le type), Disque dur

Taille logiclel: $200 \mathrm{KO}$

Langage utillsé : Turbo pascal

Imprimante conselllée : Toutes imprimantes compatibles avec l'ordinateur utilisé 


\section{Commentalre technlque :}

Appareil récent facile à programmer avec un logiciel embarqué en français.de conception et de réalisation française Permet la pH-métrie à plusieurs niveaux ainsi que la ddp. Cet appareil est le premier à présenter une fréquence d'acquisition élevée $(1 \mathrm{~Hz})$ ef un traitement du signal sur Macintosh (Hypercard) ce qui lui confére une dimension particulière. Le logiciel d'analyse est classique et performant très conversationnel. Poids et taille un peu limite pour l'utilisation pédiatrique. Utilise uniquement les sondes de verre étalonnées avant et après chaque mesure. Une idée intéressante: les accus rechargeables

\section{Commentalre de l'Expert CREGG}

Appareil à suivre présentant des innovations trés intéressante: accus rechargeable, logiciel embarqué en français, utilisation possible de Macintosh et de ses capacités graphiques, acquisition des données à une fréquence élevée qui permet la détection des micro-reflux. Il est d'utilisation simple, fiable.

\section{Commentalre général de la Commlssion :}

Appareil intéressant présentant nombre d'innovations attractives., fiable pour lequel une seule réserve concernant la taille est émise par la commission 


\section{DIGITRAPPER ED}

ABS BP 23488106 SAINT DIE CEDEX

Tél : 29584545 - Fax 29584677

Descriptlf :

Equipement de $\mathrm{pH}$-métrie ambulatoire à informatique intégrée connectable à un micro-ordinaleur PCcompatible ou directement sur imprimante

Date d'apparition sur le marché : 1990

Prix : à partir de $50000 \mathrm{~F}$ TTC

Délal de fourniture : 4 à 6 semaines

SAV et garantle : Mise en service et formation assurées par ABS.

Garantle : un an pièces et main d'oeuvre

Contrat évolutif : Evolutivité du logiciel gratuite un an

Capteur conselllé : Electrode de verre type INGOLD 440 (voire fiche spécifique) durée de vie >> $1000 \mathrm{H}$ ou éventuellement sonde à l'antimoine

Enregistreur Type convertisseur analogique-numérique transportable 1 à 2 voies, mémoire vive par voie $20 \mathrm{KO}$

Durée de vle : plusieurs années

Conflguration configurable par informatique interne

Signal digltallsé : sur 8 bits acquis à 0,5 à à, $11 \mathrm{~Hz}$ à plein usage par microprocesseur SYNECTICS Spécifique

Mobllité - Tallle - Polds : 400 Grammes, ambulatoire

Coût de fonctlonnement: 1 pile $9 \mathrm{~V}$ alcaline par examen

Système d'analyse :

Computer nécessaire : PC compatible

Type de signaux analysés $\mathrm{pH}$

Configuration fixe

Type de sauvegarde des résultats :

Disquettes ( 360 Ko à 1,44 Mo soit 10 à 40 examens) ou disque dur

Tallle loglclel: $300 \mathrm{Ko}$

Langage utlllsé : Turbo Pascal

Imprimante conselliée : EPSON LX 800 ou compatible

Coct de fonctionnement: papier imprimante 
Commentalre technique :

Appareil apparaissant en théorie (non encore essayé) intéressant par ses possibilité multiples: capable de reproduire les signaux enregistrés directement sur imprimante ou secondairement après traitement informatique sur micro. Le logiciel semble dans ce dernier cas évolué. L'appareil qui remplace le Proxima Light sera-t-il également programmable pour réaliser des mesures de Ddp?

Les seules critiques que l'on peut lui faire (d'aprés les éléments fournis) sont sa taille mémoire un peu juste (impossibilité de faire une analyse de 24 heure à fréquence d'interrogation élevée: à 0,5 $\mathrm{Hz}$ [1 point toutes les 2 secondes] 11 heures au plus peuvent être enregistrées sur chaque voies).

Des essais ultérieurs seront nécessaire pour pouvoir juger pleinement ce matériel

Commentalre de l'Expert CREGG

Appareil non expertisé non essayé.

Commentalre général de la Commission :

Appareil non expertisé par la commission. 


\section{DIgltrapper Gold MARK II}

\section{ABS BP 23488106 SAINT DIE CEDEX}

Tól : 29584545 - Fax 29584677

Descriptlf :

Equipement de $\mathrm{pH}$-métrie ambulatoire informatisé avec système d'analyse sur micro-ordinateur PCcompatible

Date d'apparltion sur le marché : 1984

Prlx : 40000 à 45000 F TTC prix Aout 1989

Délal de fournlture : 4 à 6 semaines

SAV et garantle : Mise en service et formation assurées par ABS.

Garantle : 3 ans pièces et main d'oeuvre

Contrat évolutlf : Evolutivité du logiciel gratuite un an

Capteur conseillé : Sonde Monocrystant (antimoine) durée de vie limitée à 300 heures soit environ 12 examen + référence externe $\mathrm{Ag} / \mathrm{AgCl}$ ou électrode de verre type INGOLD 440 (voire fiche spécifique) durée de vie $\gg 1000 \mathrm{H}$

Enreglstreur Type convertisseur analogique-numérique transportable lou 2 voies, mémoire vive 25 à $50 \mathrm{KO}$, par voie $25 \mathrm{KO}$

Durée de vie : plusieurs années

Conflguration fixe

Signal digltallsé : sur 8 bits acquis à $0,25 \mathrm{~Hz}$ à plein usage par microprocesseur SYNECTICS

Mobllité - Tallle - Polds : 200 Grammes, ambulatoire

Coût de fontlonnement: 1 pile $9 \mathrm{~V}$ alcaline par examen, 1 sonde antimoine + rćférence tous les $10 / 15$ examens (environ $800 \mathrm{~F} \mathrm{HT}$ ) ou 1 sonde de verre combinée (2100 F HT)

\section{Système d'analyse :}

Computer nécessaire : PC compatible

Typo de signaux analysés pH

Configuration fixe

Type de sauvegarde des résultats :

Disquettes (10 à 50 examens) ou disque dur

Tallle loglclel: $300 \mathrm{Ko}$

Langage utlllsé : Tubo-Pascal

Imprimante conselllée : toute graphique parallèle

Coct de fonctionnement: papier imprimante $400 \mathrm{~F} \mathrm{HT}$ les 2000 feuilles 
Commentaire technique :

Logiciel séduisant et performant tant sur la forme que sur le fond mais un peu desservi par un $\mathrm{pH}$ mètre aux capacités limitées: mémoire trop peu importante, manque de fiabilité des sondes à l'antimoine, sensibilité de l'appareil à des éléments externes (ondes électro-magnétiques: TV circuits électriques...). Le faible poids de l'appareil le rend aisément utilisable chez l'enfant ou la personne âgée. L'unique marqueur d'événement est simple à utiliser.

La Commission aurait aimé avoir plus de renseignement sur le type de langage utilisé pour le logiciel

\section{Commentaire de l'Expert CREGG}

L'utilisation de sondes à l'antimoine obère une partie des capacités de cet appareil dont l'impédance d'entrée a heureusement augmentée permettant ainsi une utilisation simple des sondes de verre combinées. La référence externe est source de problèmes d'enregistrement également réglés par l'utilisation de sondes combinées. La fréquence d'acquisition du signal est insuffisante masquant les phénomènes de courte durée. Par contre le logiciel de traitement est trés convivial et performant permettant aussi bien un travail de routine qu'un travail de recherche.

Commentaire général de la Commission :

Appareil léger soutenu par une bonne informatique, pour lequel il faut privilégier l'utilisation des sondes de verre. Une augmentation des performances (taille mémoire et fréquence d'interrogation) serait la bienvenue sur cet appareil dont la conception date déjà un peu. Service après vente inégalement apprécié par les utilisateurs 